S. Stoyanov, C. Bailey and G. Tourloukis (2016) "Similarity approach for reducing qualification tests of electronic components", Microelectronics Reliability, Volume 67, December 2016, pp. 111119

Link to published version of the paper:

https://doi.org/10.1016/j.microrel.2016.10.017 


\title{
Similarity Approach for Reducing Qualification Tests of Electronic Components
}

\author{
Stoyan Stoyanov, Chris Bailey and Georgios Tourloukis \\ Computational Mechanics and Reliability Group, University of Greenwich, London SE10 9LS, UK \\ Email: s.stoyanov@gre.ac.uk
}

\begin{abstract}
Qualification analysis and reliability testing of electronic components represent a major activity in the process of development of electronic equipment. Electronics manufacturers have to adopt often costly test programmes to ensure qualification standards and reliability requirements are met. This paper details a novel similarity-based qualification approach for assessing expected reliability of electronic components, and in general electronic products, as an alternative to conventional physical testing. The originality of this work is in the proposed approach which introduces a new way of qualifying electronic components based on similarity with previously assessed and qualified components. This novel approach has the potential to transform in a major way the current operational practices in the industry, demanding at present substantial physical testing, by offering a complementary, "virtual qualification" route for addressing the cost and time challenges associated with qualification tests.

A major achievement is the comprehensive and detailed demonstration of the proposed reliability qualification approach. Case studies on qualifying electronic components to the thermal loads induced by a particular post-manufacturing process widely adopted by high-reliability electronics sectors - the robotic hot solder dip (refinishing) process, and associated risks of thermo-mechanical damage are presented and discussed.
\end{abstract}

Keywords: similarity analysis of electronic components; reliability qualification tests; hot solder dip process; self-organizing map

\section{INTRODUCTION}

Diverse range of new commercial-of-the-shelf (COTS) electronic components are constantly developed and delivered to the market. In this paper, the terms component and/or part refer to the associated package of silicon-based circuits. Qualifying new electronic components within increasingly shorter design development cycles and increased test requirements becomes a major challenge for many industries. There is a strong need for alternative qualification approaches to conventional physical testing of electronic components and products that can substitute, where appropriate, costly reliability test programmes.

The electronics industry follows broadly two main approaches to reliability qualification: (1) Stressbased approach and (2) Knowledge-based approach [1]. With the former approach, the qualification assessment is prescribed and aims to evaluate the capability of an electronic product to pass a specified set of stress test procedures. This approach is therefore known as "stress-based" or "standards-based". Stress-based qualification procedures are documented, standardised tests that define accelerated load conditions, usually in terms of temperature, relative humidity, duration, etc. The knowledge-based, or "use conditions"-based, qualification adopts customised tests that rely on knowing and understanding the failure mechanisms of the technology and applying usage environments as part of the qualification plans. Knowledge-based qualifications are superior compared to standard-based methods as they seek to incorporate failure mechanism-based 
methodologies and encompass additional information about the design, technology and the manufacturing processes [1].

Computational intelligence algorithms have been subjected to a growing interest in recent years $[2,3]$ and used extensively in many applications such as pattern recognition, system modelling, intelligent control problems, fault identification, biomedical etc. [4]. Similarity-based methods in particular are among those that have been well researched $[2,5]$. For example, the Self-Organising Map, an unsupervised neural network model used in this study, has found numerous applications in engineering including in the monitoring, fault diagnostics and identification of the states of manufacturing equipment and processes with complex behaviour [6-8]. The SOM is a model capable to perform projection of non-linear high dimensional data of input sensed signals or other distinctive characteristics onto low-dimensional data map in ordered manner $[2,12]$.

The problem of using structural similarity as a method to reducing qualification tests of IC and electronics package designs, and wafer fabrication has been studied by van Driel et al. [9-11]. Their work marks a substantial effort in overcoming the shortcomings of some traditional similarity-based rules typically deducted from past experience and trial-and-error results. This study developed the so called structural similarity assessment in the form of Design-of-Simulations based response surface model that authors then use to evaluate performance/reliability of a new product and used the model predictions to make decisions if qualification tests are required. As such, this approach does not actually qualify a product based on similarity to other previously qualified products but rather makes this decision based on assessing quantitatively specified responses through developed numerical surrogate models. This approach requires substantial effort in defining and preparing the design-of-experiments parametrised physics-of-failure model and undertaking the associated computational analyses.

While model-based qualification of electronic components is advised and is possible in most cases, and despite of all advantages over experimentation, developing and using detailed models to evaluate behaviour and responses of electronic parts when subjected to process/test conditions and loads may still require substantial effort. The interesting question is, can detailed modelling work for qualifying electronic components be avoided in certain circumstances and when?

The capability and potential usage computational intelligence in enabling similarity-based qualification of electronic components is not yet recognised. In many engineering applications behavioural, operational, performance, reliability, etc. aspects of products or processes related to design, manufacturing and use can be anticipated based on existing past knowledge for "similar" designs and processes. In the case where products have to meet user or standard related reliability specifications and requirements, similarity analysis can aid qualification of new products on the basis of sufficient similarity with previously qualified products. Before similarity can be observed, evidence generated through experiments or models for the effect of a selected attribute and its relationship to critical test outcomes must be generated.

While "similarity-based qualification" is clearly based on knowledge and hence at high level can be seen as knowledge-based, this approach has also some fundamental differences with respect to the generic "Knowledge-Based Qualification". Knowledge-based Qualification, unlike the standardsbased qualification approach, aims to develop first the knowledge about the specific technology/ product failure mechanisms and then to use the knowledge in adopting tailored conditions-based qualification plans. Although the approach is knowledge-informed, it still relies on using relevant experimental tests for reliability qualification. Knowledge-based qualification is about a smarter and more representative for the use conditions way of defining better qualification tests (shorter tests, using fewer parts, improved in-service prediction of reliability). The similarity approach presented in the paper seeks to eliminate, where appropriate, experimental and/or complex physics-of-failure model-based reliability evaluations and instead use product attributes affecting failure risks (with respect to anticipated failure modes) to draw conclusions about its expected qualification status. 
The similarity approach is applied assuming the qualification test plan is decided - hence it can be used within both a standards-based and knowledge-based qualification frameworks. The approach of similarity-based qualification implies qualifying the parts to specific process without subjecting the parts to respective qualification experimental tests.

Building upon this notion, the demonstration of the developed similarity approach for reliability qualification of electronic components focuses on a particular post-manufacturing process termed hot solder dipping. Hot solder dipping, also known as refinishing, process is predominantly adopted by high reliability electronics manufacturer as risk mitigation strategy against tin whiskers related failures [18].

\section{COMMON SIMILARITY MODELS}

Similarity can be seen as the relationship that holds between two objects. It can be considered as the perceptual resemblance of objects to one another. In the context of this research work, an object is an electronic component. There are different approaches to similarity analysis and common similarity model types, for example:

1. Common element models

This approach is suitable where objects naturally can be represented by different elements. Similarity is calculated counting the number of common elements relative to other elements and/or by summing their values.

2. Template models

These models rely on spatial point-for-point comparison between the objects. The degree of correspondence determines the level of similarity.

3. Feature models

This is the most common approach where objects are represented by set of features (or attributes). The central assumption is that the similarity between two objects $\boldsymbol{a}$ and $\boldsymbol{b}$ is that it is a function of the features common in the two objects, those in $\boldsymbol{a}$ but not $\boldsymbol{b}$, and those in $\boldsymbol{b}$ but not in $\boldsymbol{a}$. Similarity with these models is not just a function of common features but it depends also on features that are unique to each object.

4. Geometric models

Similarity relationships are represented among objects and a "similarity space". Similarity is defined by distance between objects in this space. The closer in terms of the distance between them two objects are the more similar they are. The similarity space with geometric models is typically represented by attributes of objects related to geometry (e.g. size) and other measurable attributes. These models are most common in similarity analysis.

For the purpose of similarity-based qualification of electronic components, a suitable approach is to adopt an attribute-based (or feature-based) classification of the components combined with a common distance measure evaluation in the defined similarity space. In computational similarity algorithms, the attributes (features) are represented typically in a vector format.

The proposed similarity approach in this work falls within the domain of similarity-based classification. Similarity-based classifiers estimate the cluster (group) label for an object based on the similarities between the object of an interest and a set of already clustered objects. This process involves two main steps: (1) generating a set of clusters, or categories, and (2) classifying given objects into the created categories. 
Similarity based classification can use different algorithms from science domains such as computational intelligence, machine learning, data mining, etc. [2,3]. The computation of similarity uses typically some form of a distance measure. Examples of commonly used distance measures in similarity algorithms include the squared Euclidian distance and the Radial Basis Function (RBF) kernel although other options also exist. For two objects represented with their respective attribute/feature vectors $\boldsymbol{x}$ and $\boldsymbol{y}$, these two distance measures are computed as:

- Squared Euclidean distance, $d^{2}(x, y)$ :

$$
d^{2}(x, y)=\|x-y\|_{2}^{2}=\left(x_{1}-y_{1}\right)^{2}+\left(x_{2}-y_{2}\right)^{2}+\ldots+\left(x_{n}-y_{n}\right)^{2}
$$

where $n$ is the number of attributes

- $\quad$ RBF kernel, $K(x, y)$ :

$$
K(x, y)=e^{-\frac{\|x-y\|_{2}^{2}}{2 \sigma^{2}}}
$$

where $\sigma$ is a "free" parameter.

\section{COMPUTATIONAL INTELLIGENCE APPROACH TO SIMILARITY CLASSIFICATION}

\subsection{Self-organising Map (SOM) Algorithm}

SOM is a neural network with nodes organised in two layers only: (1) an input layer where each node takes the input value of one of the attributes upon which the similarity classification is decided, and (2) the output layer. The process of classification based on similarity involves projecting (mapping) the $n$-dimensional input data space (the attribute values for the input) to the discrete output space, a two-dimensional grid of elements (clusters) known as a feature map. The method clusters the input data while maintaining the topological structure of the data in terms of similarity. The outputs have the meaning of similarity classifiers of the input data. The major advantages of the SOM are its capability to self-learn and self-adapt when exposed to new data.

In a SOM network, each element on the feature map is associated with $n$-dimensional weight vector ( $w_{j}$ where $j=1$ to $\mathrm{m}, \mathrm{m}$ being the number of feature map elements) that forms the centroid of one cluster. Similarity is measured by the SOM elements in the output layer using Euclidian distance between the network weight vectors associated with all cluster elements and the input data vector. The element with the minimum distance (most similar) is the winner and outputs a one to the output layer; all other elements are forced to zero as this is a competitive network model. The winning element is therefore nearest to the input value - this element (cluster) represents the input in the two-dimensional map. The training of SOM involves stochastic training rules: the weight vectors are updated using all input data available in an iterative manner until convergence criteria are met [2].

Figure 1 shows an example of a network with $n$ inputs for SOM with output layer of $3 \times 3$ elements. 


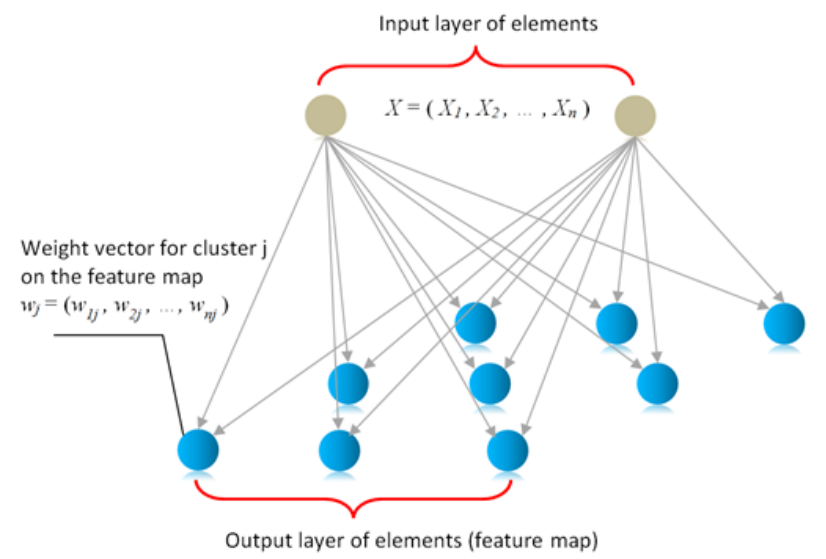

Figure 1: Example of self-organising map neural network model with $n$ inputs and 9 clusters (feature map with $3 \times 3$ nodes in the output layers).

The data points presented to the similarity-based clustering algorithm are in the form of $\boldsymbol{n}$ dimensional vectors. The SOM is established through unsupervised training process using training data set of input vectors. The size of the SOM feature map can be generally defined as $N \times M$ but in most cases $N \times N$ grid is used. The total number of clusters in the feature map is assumed to be $\boldsymbol{m}$ ( $\boldsymbol{m}$ typically of order $5 \sqrt{P_{T}}$ where $P_{T}$ is the number of the training vectors).

The training of a SOM network model is realised through an iterative process. The cluster that is closest to the input vector is called Best Matching Unit (BMU). This BMU weight vector is denoted with the index $c$ and can be written as

$\mathbf{w}_{c}=\left(w_{1 c}, w_{2 c}, \ldots, w_{n c}\right)$

Once the BMU is identified at the particular training step (i.e. at time $t$ ), the weight vectors of the $\mathrm{BMU}$ and its topological neighbouring clusters are updated so that they all move closer, to different degree, to the input vector. As a result, the BMU and the associated topological neighbours adapt to be closer to the sample vector selected at the current training step [14, 15].

The generic SOM update rule for the weight vector $\mathbf{w}_{j}$ of a cluster $\boldsymbol{j}$ can be expressed as

$$
\mathbf{w}_{j}(t+1)=\mathbf{w}_{j}(t)+\alpha(t) h_{c j}(t)\left[\mathbf{x}(t)-\mathbf{w}_{j}(t)\right]
$$

where $t$ denotes the time associated with the current training step, $\mathbf{x}(t)$ is the randomly selected input vector from the training dataset at time $t, \alpha(t)$ is the time decaying learning rate at time $t$, and $h_{c j}(t)$ is the neighbourhood function around the BMU (cluster $c$ ). The neighbourhood function is a decreasing function of time and the distance between the BMU and the cluster with index $i$. As an example, a possible choice for the $h_{c j}(t)$ is the so-called EP-Kernel function defined as

$$
h_{c i}(t)=\max \left\{0,1-\left(r(t)-d_{c i}\right)^{2}\right\}
$$

where $r(t)$ is the neighbourhood radius at time $t$ and $d_{c i}$ is the distance between clusters $c$ and $\boldsymbol{i}$ on the 2D feature map (based on cluster's grid coordinates and not the cluster weight vectors).

The generic steps of the SOM algorithm when using sequential training procedure, in the format of a pseudo-code, are detailed in Figure 2. 


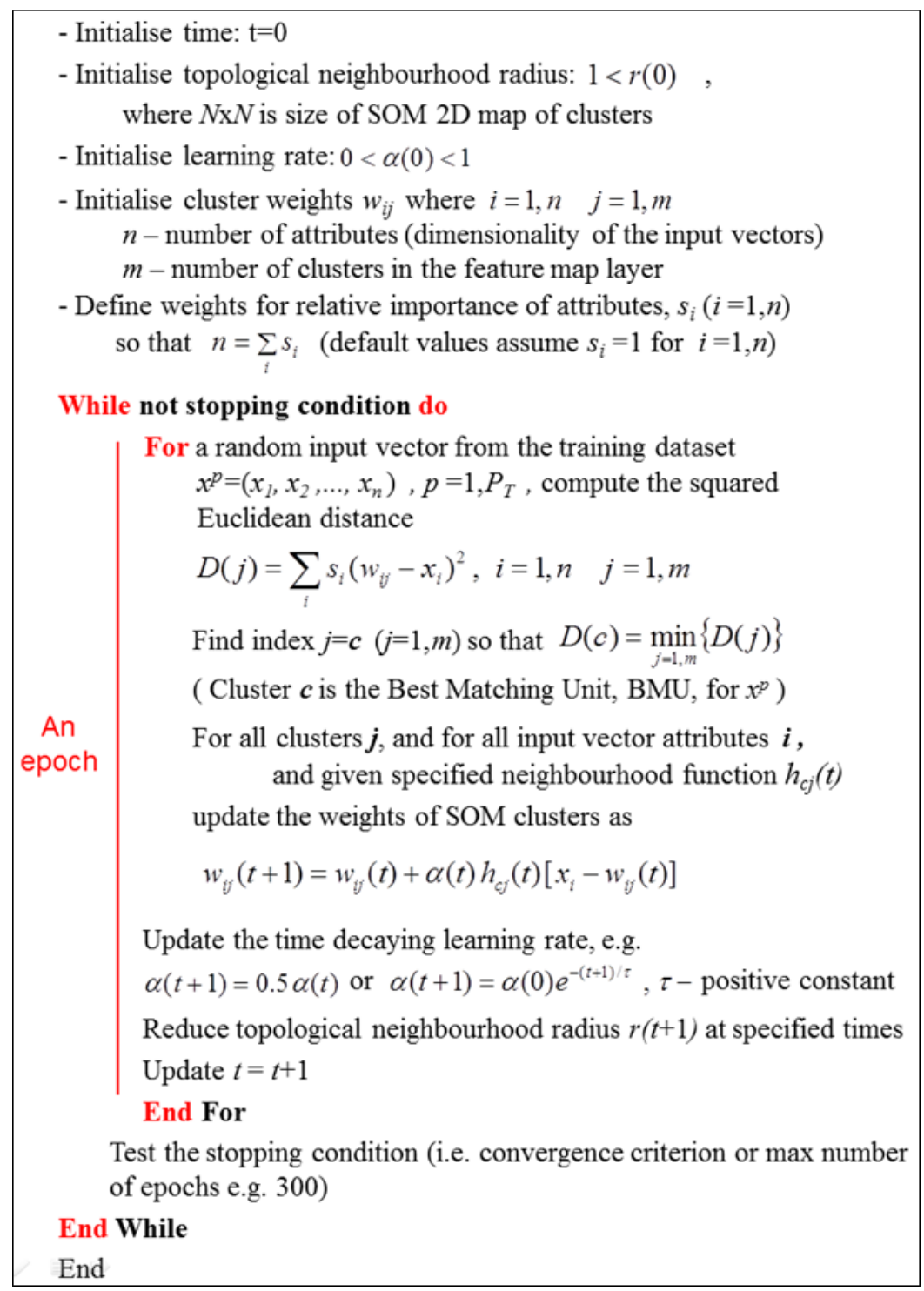

Figure 2: Pseudo-code of SOM training algorithm.

There are different realisations of the SOM algorithm with respect the initial settings of parameters and the choice of the training procedure. For example, it is possible to preform so called "batch" training instead of the outlined above sequential iterative training. Details about the "batch" model of training can be found in reference [14].

\subsection{Assessment of Model Accuracy}

The quantisation error is an indicator for the map accuracy and measures the SOM feature map resolution. It is computed as the average distance between each input data vector and its respective $\mathrm{BMU}$. At training step $t$, the quantisation error is defined as: 


$$
\varepsilon_{T}=\frac{\left(\sum_{p=1}^{P_{T}}\left\|x^{p}-w_{c}^{p}(t)\right\|_{2}^{2}\right.}{P_{T}}
$$

where $\boldsymbol{c}$ is the index of the BMU cluster $\left(\mathbf{w}_{c}{ }^{p}\right)$ for input vector $\mathbf{x}^{p}, \mathbf{x}^{p}$ is an $\mathrm{n}$-dimensional input data vector, and the number of the input vectors is $P_{T}$. Training can stop when $]_{T}$ is sufficiently small.

The topographic error is the proportion of all data vectors for which first and second BMUs are not adjacent clusters on the feature map. Hence, this error is a measure of the degree to which the topology of the input data is preserved when this data is mapped onto the feature map.

Both these measures give better results when the map is over fitted to the input data, i.e. when the number of the clusters in the feature map is larger than the number of the training input vectors. In such cases, obtaining a SOM with both of the errors equal to zero should be avoided.

\section{RATIONALE FOR SIMILARITY BASED RELIABILITY QUALIFICATION}

The availability of efficient computational techniques for performing similarity analysis has the potential to offer a complementary approach to physical testing, where this is appropriate, in relation to reliability qualification of electronic components. The principle behind similarity-based reliability qualification builds upon the notion that if a new component that needs to be assessed is sufficiently similar to components which have been already qualified in the past, it can be expected that the new component will exhibit very similar behaviour and response characteristics to the same process/tests loads and conditions. Hence the qualification status of the previously qualified parts can be projected onto the new part, i.e. the new part can be qualified based on similarity, and will therefore not require a complete physical testing.

The main challenge with this approach is the definition of relevant set of attributes of the electronic components upon which the similarity classification should be performed. The selection and definition of the attributes would require prior knowledge what characteristics/parameters of the electronic components impact most the anticipated failure modes and mechanisms. Such attributes can therefore be seen also as vulnerability parameters, i.e. parameters that indicate susceptibility to damage with respect to defined failure mechanism. If the attributes can be defined comprehensibly and with confidence then similarity of two components on the basis of these attributes will imply similar reliability risks under defined process, qualification test or operational conditions, and hence no real physical tests would be required. In the case of reliability qualification, this brings the advantage of reducing the number of parts requiring physical testing and hence result in time and cost effective qualification process.

Susceptibility to damage of an electronic component when subjected to load conditions from process, test or operation depends predominantly on constructional design, materials and manufactured quality as well as other factors. For this reason, similarity attributes in the context of this similarity analysis requirements need to capture and account for key dimensional characteristics and constructional and material relations of the electronics component that influence the reliability risks under the specified load conditions. 


\section{QUALIFICATION REQUIREMENTS FOR REFINISHING OF ELECTRONICS COMPONENTS: AN APPLICATION}

In this study, the potential of using similarity-based approach with SOM to qualification and reliability assessments of electronic parts is demonstrated in the case of hot solder dip refinishing process [16-18]. This post-manufacturing process is adopted at present by many electronics manufacturers of equipment used in high reliability applications as a tin-whisker risk mitigation strategy [19]. With this process, lead-free finishes on component terminations are replaced with tinlead solder by dipping the leads of the part in tin-lead molten solder bath. As this process involves exposure of the part to a thermal load from hot solder dipping, there is also potential risk of inducing thermo-mechanical damage. Qualifying an electronic part as undamaged after refinishing using hot solder dip process is not trivial and at present is addressed through performing a series of experimental qualification tests [20].

Validated thermo-mechanical models of double dip hot solder dip process have been developed in the past and shown very good predictive capabilities $[17,21]$. These modelling methodologies and the developed models are not discussed here but are referenced because they are used to define and evaluate the effects of a set of vulnerability parameters on the susceptibility to damage of leaded components to hot solder dip thermal loads. In this discussion, the part's failure mechanism and associated failure mode of interest in the context of part qualification to applied hot solder dip thermal loads is delamination the interface between the package moulding compound and the dipped side of the lead-frame caused by the difference in the coefficient of thermal expansion on the respective package materials.

As an example, Figure 3 (a) illustrates two leaded packages and their internal constructions obtained using 3D CT scan package characterisation. The associated finite element model predictions for stress intensity at the package lead-frame to moulding compound interfaces, illustrated in Figure 3 (b), come from a simulations of the respective package thermo-mechanical response to a hot solder dip load. The hot solder dip load is induced as a result of dipping the leads at one package side into modern solder with temperature $250^{\circ} \mathrm{C}$ for duration of 3 seconds. The stress intensity level at the observed package bi-material interface determines the risk level for delamination damage caused by the applied thermal load.

Figure 4 shows an example of the trend in stress alternation as function of a particular vulnerability parameter defined as the ratio of the area of the IC die to package thickness. A parameter is indicative for vulnerability if it has a clear effect on the respective failure mechanism. For example, larger value of the "die area to package thickness ratio" is associated with larger interfacial stresses and hence increased risk of delamination damage. In this instance the thermo-mechanical simulation results suggest that the stress intensity can increase by as much as $0.03 \mathrm{MPa}$ per $1 \mathrm{~mm}$ change in the "die area to package thickness ratio" (see Figure 4).

The vulnerability parameters presented in this work are straightforward to calculate for an electronic component that has undergone package constructional and material characterisation. These vulnerability parameters can be seen also as attributes of the electronic component. For this reason, vulnerability parameters are the natural candidates to consider and use as inputs for a similaritybased clustering algorithm. 

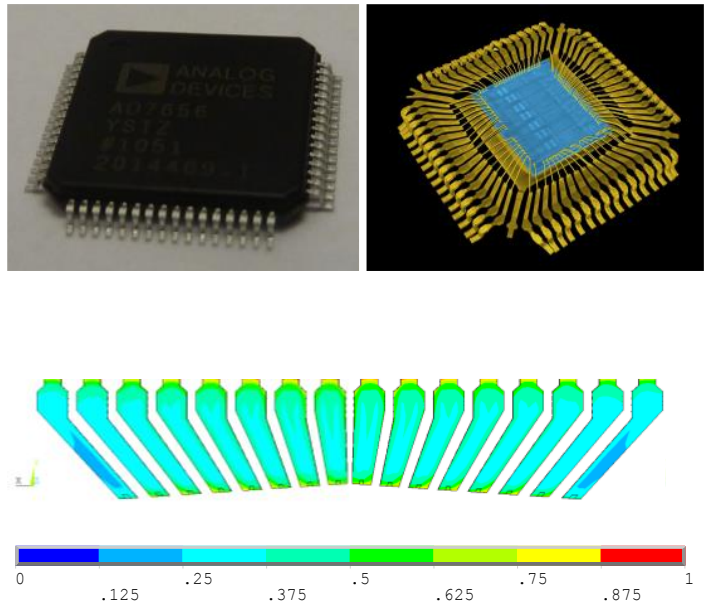
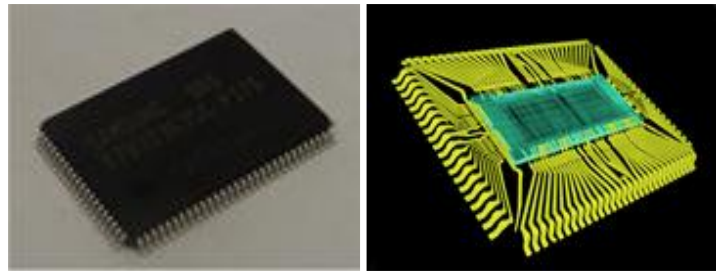

(a)

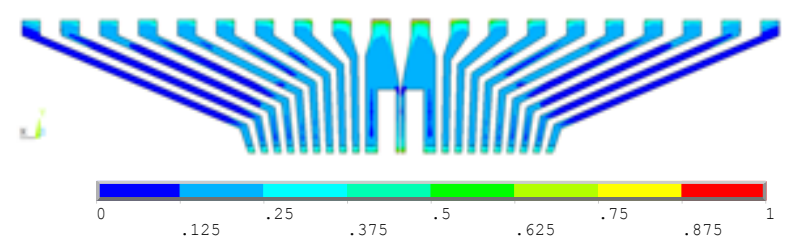

(b)

Figure 3: (a) Two typical QFP-type component constructions and (b) associated examples of FEA predicted stress intensity contours (normalised) at the interface of the lead-frame with the moulding compound at the hot solder dipped package side (Thermal load of $250^{\circ} \mathrm{C}$ for $3 \mathrm{sec}$ )
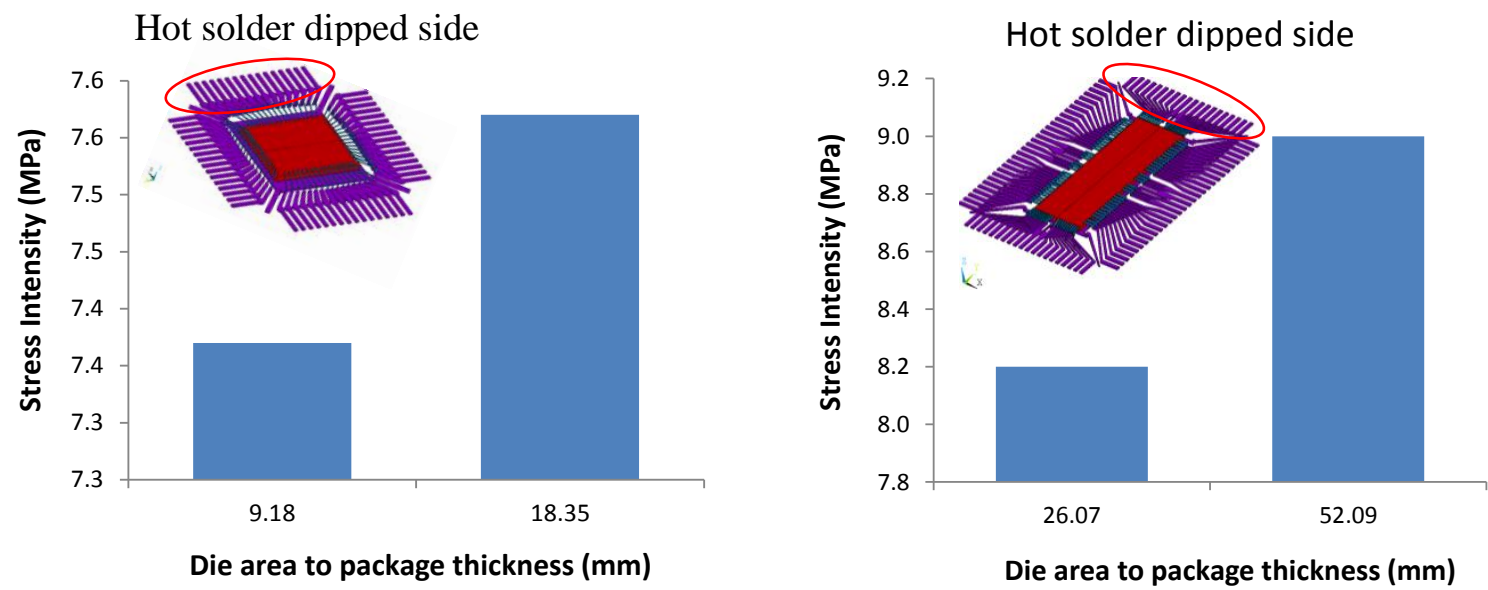

Figure 4: Stress intensity level at the lead-frame and moulding compound interface for two QFP generic components as function of a vulnerability parameter value.

Assuming typical refinishing process conditions (solder bath at $250^{\circ} \mathrm{C}$ and single side dipping for 3 sec), the following vulnerability parameters for leaded QFP-variant package types in relation to hot solder dip thermal loads have been formulated:

1. Die area to package thickness. Larger values of this vulnerability parameter are associated with higher risks for thermo-mechanical damage. In general, larger silicon die in thinner package makes the coefficient of thermal expansion (CTE) miss-match between the package materials more severe, and hence increases susceptibility to damage under applied thermal loads

2. Die area to package area. Larger values of this vulnerability parameter are associated with higher risks for thermo-mechanical damage. This parameter complements the vulnerability assessment trough the parameter "die area to package thickness" as it evaluates the die size in the context of the package size.

3. Dipped terminations cross section area to package volume. This parameter is specific for the refinishing process application. It is an indicator of the amount of heat transferred into the 
package during dipping the terminations in molten solder for fixed dip duration. Larger values of this vulnerability parameter mean higher temperatures are reached in the package internals and hence larger thermal loads. This is associated with increased risks for thermo-mechanical damage at package level and can also affect IC characteristics.

4. Moulding compound (MC) thermal conductivity. This property of the moulding compound affects the temperature distribution in the package due to the applied refinishing process thermal load, particularly the maximum temperature of die level. The parameter has less influence on the susceptibility of the package to the delamination damage mode considered in this study.

5. Moulding compound coefficient of thermal expansion CTE. This material property has major impact on the degree of CTE mismatch between the package materials. As the solder dip loads are associated with temperatures above the glass transition temperature $(\mathrm{Tg})$ of the moulding compound, moulding compounds with lower CTE above the Tg match better the CTEs of the rest of the package materials and hence indicate lower chance for thermo-mechanical damage.

The study on derivation and formulation of the listed vulnerability parameters, and how they impact a set of package thermo-mechanical responses related to common failure modes is subject of a separate publication. At present this study can be referenced with a project report on this investigation [13]. Particular thermo-mechanical simulation results that are used in the next section of the paper refer to the model predictions from a parametric study on each of the above stated attributes. The modelling results evaluate the magnitude of stress intensity change when an attribute changes from a lower to upper limit over a typical range of the attribute values. This information is provide in the next section where both the range of the attribute and the maximum alternation in the stress over the respective attribute range are listed.

\section{DEMONSTRATION OF SIMILARITY-BASED QUALIFICATION OF ELECTRONIC COMPONENTS}

The study case refers to the problem of qualifying quad-flat package (QFP) type electronic components to typical hot solder dip thermal load condition. Given the formulation of several vulnerability parameters that can indicate varying risks for damage, these parameters are used as the actual component attributes upon which the similarity assessments are performed. With this approach, new attributes that capture other component aspects affecting component behaviour and response can be formulated with no limitation and added to the list of attributes. In this demonstration it is assumed that a selection of 36 QFP-type components is fully characterised in terms of the specified five attributes and have known qualification status decided upon respective thermo-mechanical responses (delamination damage or no damage) of the parts to the refinishing process loads. The attributes of the assessed parts and their ranges are summarised in Table 1 . Note that all calculations reported in the remaining sections of the paper are based on values of the attributes in the normalised attribute design space (0-1 range).

The last column of Table 1 captures the model-based predictions for the linear (main) effect of each of the attributes on the stress intensity parameter affecting the package delamination risks. 
Table 1: Definition of similarity attributes for quad-flat package type components in relation to their susceptibility to thermo-mechanical damage due to hot solder dip thermal loads.

\begin{tabular}{|l|c|c|c|c|}
\hline \multicolumn{2}{|c|}{ Attribute } & Attribute Range & $\begin{array}{c}\text { Normalised } \\
\text { Range }\end{array}$ & $\begin{array}{c}\text { Signed value of } \\
\text { maximum change in } \\
\text { stress intensity } \Delta S_{\text {int }} \\
\text { (MPa) over attribute } \\
\text { range (low to high } \\
\text { level) }\end{array}$ \\
\hline $\begin{array}{l}\text { Die area to package } \\
\text { thickness (mm) }\end{array}$ & a1 & $9.0-52.0$ & $0-1.0$ & 1.32 \\
\hline Die area to package area (\%) & a2 & $6.0-25.0$ & $0-1.0$ & 1.00 \\
\hline $\begin{array}{l}\text { I/O cross section to package } \\
\text { volume (10-2 } \mathbf{m m}^{-1} \text { ) }\end{array}$ & a3 & $0.10-0.27$ & $0-1.0$ & 0.33 \\
\hline $\begin{array}{l}\text { MC CTE (ppm/K) } \\
\text { MC thermal conductivity } \\
\text { (W/mK) }\end{array}$ & a5 & $\begin{array}{c}8.0-12.0 \text { below Tg } \\
24.0-35.0 \text { above Tg }\end{array}$ & $0-1.0$ & 2.46 \\
\hline
\end{tabular}

\subsection{Constructing the SOM Classifier}

In this demonstration the set of the 36 previously qualified (through physical testing) components is used to build the SOM similarity classifier. Each part is represented by a five-dimensional vector of the defined attributes in the normalised space as defined in Table 1. A cut-off list of the parts is given with Table 2.

Table 2: Example of components forming the list of 36 previously assessed components with known qualification status.

\begin{tabular}{|l|l|l|l|l|l|}
\hline \multirow{2}{*}{ Part No. } & \multicolumn{5}{l}{ Normalised Attribute Value } \\
\cline { 2 - 6 } & a1 & a2 & a3 & a4 & a5 \\
\hline Part No. 1 & 0.427 & 0.318 & 1.000 & 1.000 & 0.240 \\
\hline Part No. 2 & 0.284 & 0.000 & 0.068 & 0.615 & 0.200 \\
\hline$\ldots \ldots \ldots .$. & 0.873 & 0.373 & 0.731 & 0.065 & 0.832 \\
\hline Part No. 35 & 0.211 & 0.263 & 0.353 & 0.458 & 0.524 \\
\hline Part No. 36 & & & & & \\
\hline
\end{tabular}

The SOM model is developed using the MATLAB integrated SOM toolbox [14, 15]. The 36 parts form the training data set used to construct the SOM. The distance measure used in the classifier in this instance is the Euclidian distance. The SOM selected with this demonstration has a feature map with cluster grid size $6 \times 6$. The SOM grid is illustrated in Figure 5 . 


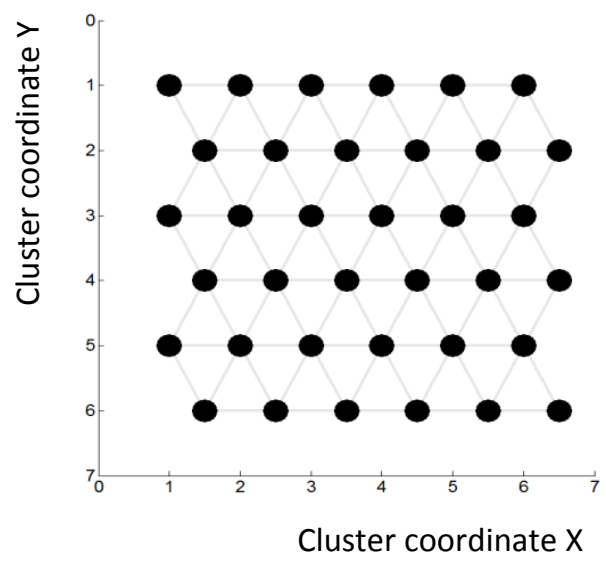

Figure 5: SOM feature map.

The training of the SOM is executed in "batch" mode. The initial (before training) weight vectors assigned to clusters are illustrated with Figure 6 . This graph visualises the scaled values of both the SOM weights (black dots) and all 36 training data vectors (red crosses) in the two-dimensional space of the first two attributes ( $a 1$ and a2). Similarly, Figure 7 shows the weight vectors values in the trained SOM. This is after iterating over 300 epochs. On a desktop computer with Intel Xeon CPU at $3.10 \mathrm{GHz}$ and $128 \mathrm{CB}$ RAM, the computation time associated with the SOM network structure definitions, training dataset processing and the actual training (300 epochs) of the network resulting in a constructed SOM model takes approximately 20 seconds.

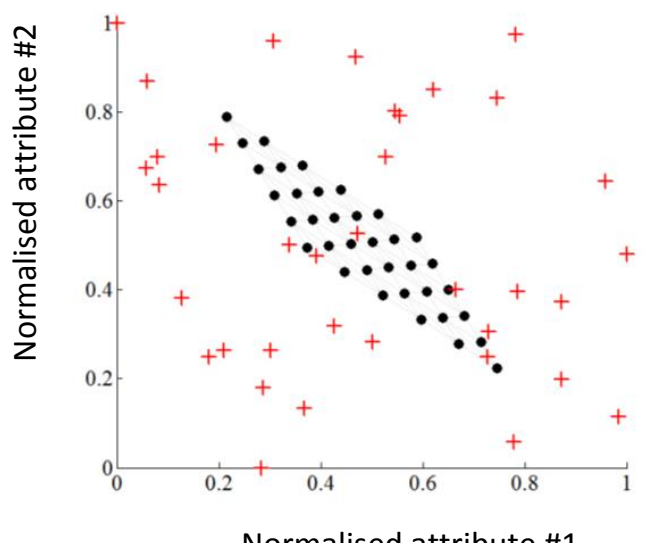

Normalised attribute \#1

Figure 6: Initial (before training) SOM weights (black dots) and training input vectors (red crosses) in the 2-dimensional space of attributes \#1 (a1) and \#2 (a2). 


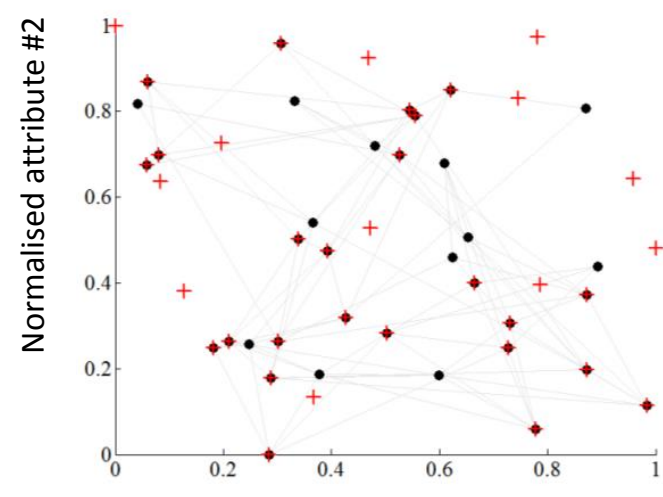

Normalised attribute \#1

Figure 7: SOM weights (black dots) after training with input vectors (red crosses) in the 2dimensional space of attributes \#1 and \#2.

The quantisation error at the end of the batch mode training process with 300 epochs is 0.0683 and the topographic error is 0.0833 . The latter error indicates that only 3 out of the 36 training input vectors have their first and second BMU clusters not adjacent on the feature map. Both errors indicate that the quality of the SOM in terms of distance deviation of input data vectors from clusters is very small and the overall preservation of data topology (defined as the neighbourhood) is also good. Figure 8 shows the history of the quantisation error at each epoch during the iterative SOM training.

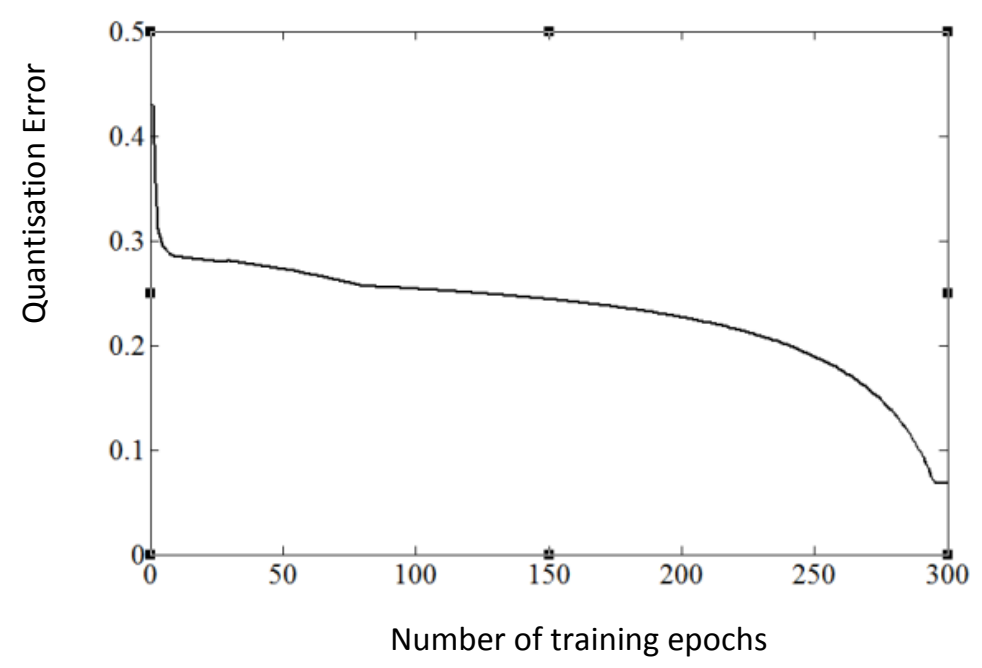

Figure 8: Change in quantisation error for SOM quality with training epochs.

Once the classifier is trained, it is possible to consider the so called hits diagram. This shows, as illustrated in Figure 9, the hexagonal grid of SOM clusters, with their existing topological relations and ordering, and for how many input data vectors each cluster has become the respective BMU. The algorithm implementation allows to output results what input data vectors are mapped onto what clusters. 


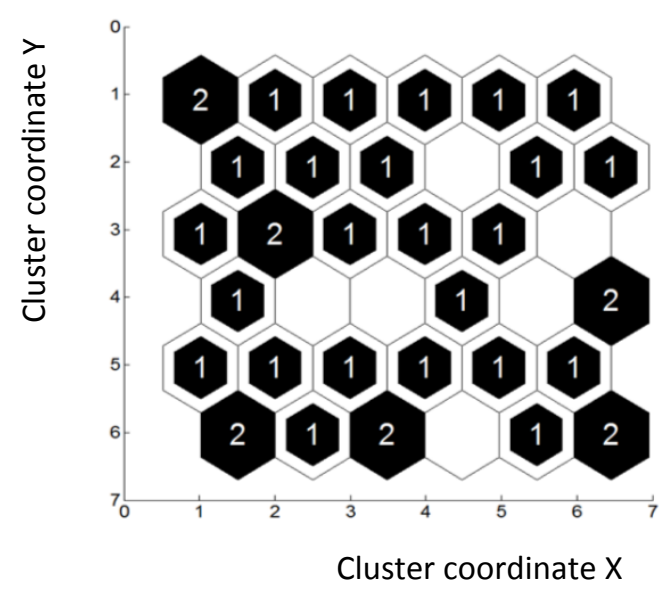

Figure 9: SOM data histogram (hits) showing number of input vectors mapped onto each cluster.

\subsection{Similarity Index Definition}

While similarity-based classifiers such as SOM have excellent capability to cluster high-dimensional input data and hence identify through BMU the most similar previously assessed (or qualified as with this application) object to a new object of interest, the level of similarity is not explicitly known. Therefore, it is useful in addition to the classifier's output results to have also an indicator for the degree of similarity.

In the context of the demonstration study, the use of a similarity index $(S /)$ is proposed. The objective is to use the $S I$ value in relation to a set threshold limit to decide if similarity level is sufficient to consider qualification of the new electronic component on the basis of similarity to the BMU parts only, or if qualification (physical testing, etc.) will be required. In the case of SOM, the feature map resolution is a model characteristic that affects the distance between the new input data vector and its BMU. However, for a given set of training data vectors SOM resolution will not affect the distance between the two most distant (dissimilar) clusters on the feature map. Therefore, an $S I$ that is representative of the observed similarity between a new part and its BMU in relation to the distance between the most dissimilar map clusters is considered. The proposed $S /$ is defined as:

$$
S I(\%)=\left\{\begin{array}{cl}
\left(1-\frac{D_{B M U}}{D_{\max }}\right) 100 & , \text { if } D_{B M U} \leq D_{\max } \\
0 & , \text { if } D_{B M U}>D_{\max }
\end{array}\right.
$$

where $D_{B M U}$ is the distance from the new part to its BMU cluster on the feature map, $D_{\max }$ is the maximum distance between any two clusters in the SOM map. The similarity index is calculated in \%, with higher values indicating higher level of similarity.

The definition of a threshold limiting value (TLV) for the SI index is application specific and would require applying good engineering judgement and failure mechanism knowledge. The guiding principle is to define the TLV so that it reflects existing knowledge on the failure mechanism sensitivity to attribute changes.

\subsection{Similarity-based Classification Study Case}

A new electronic component labelled $A$, with normalised attribute vector

$$
\mathrm{X}_{A}=(0.440,0.330,0.969,0.954,0.252)
$$


is presented to the SOM classifier developed in Section 6.1. The algorithm finds the best matching unit (BMU) for the new part as cluster \# 2. The locations of the new component and the weight vector of the BMU, as they appear on the SOM feature map, are shown in Figure 10.

The user can then make reference to the cluster definitions of the SOM. In this case, cluster \#2 has 1 hit which means that during training only one of the training input vectors associated with the 36 previously qualified parts is mapped onto this cluster. This part is the one labelled as No.1 in the training set (see Table 2 ) and the cluster weights $\left(w_{2}\right)$ have the same values as the values of the five attributes of this part. The clustering results from SOM informed that the most similar previously assessed part to the new is part No.1. It can be verified that the new part A has attributes that differ by approximately $2-3 \%$ from the attribute values of part No.1.

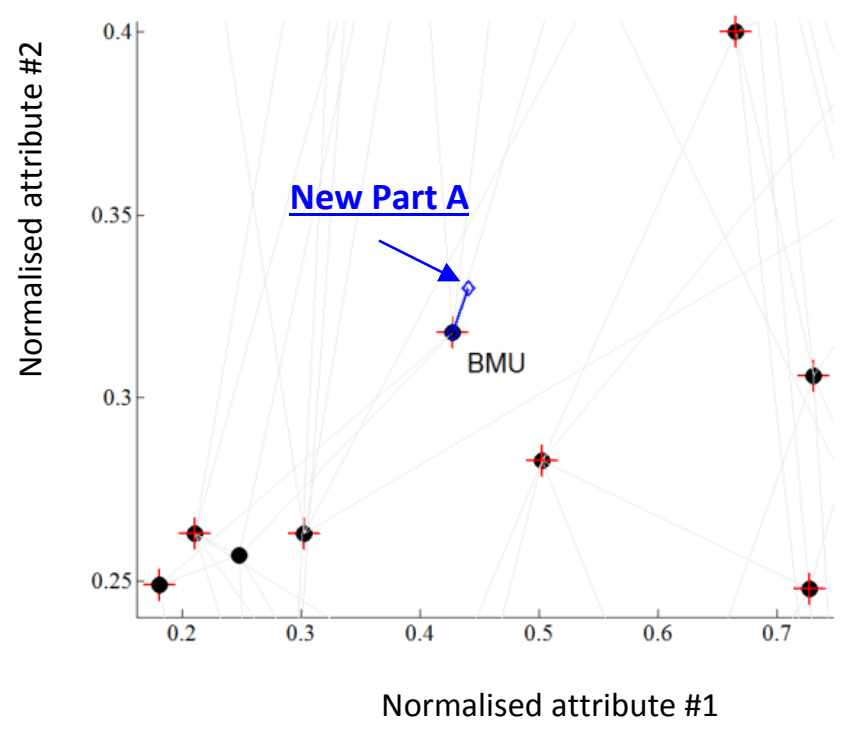

Figure 10: New Part A and its BMU cluster (\#2) in the two-dimensional space of attributes \#1 and \#2.

The largest distance between the two most dissimilar clusters in the SOM map is 1.62. These are clusters \#1 and \#36. Note that SOM algorithm has managed successfully to preserve in the output layer of clusters the topology of the input data vectors. Given that the distance between the new part $A$ and its BMU is 0.0594 , the similarity index $S /$ is found as $96.3 \%$. This can be compared in relation to a set threshold value, and on the basis of this additional criterion to decide if the new part can be qualified through its similarity to part No.1.

Consider now a new part B notably different than any of the original 36 parts used for the SOM training. The attribute values for new part $B$ are also outside the design space limits given with the training data vectors. The new part $B$ has the following (normalised) values of attributes:

$$
X_{B}=(1.287,1.152,1.339,-0.077,1.400)
$$

The algorithm finds the best matching unit (BMU) for the new part $B$ as cluster \# 29 . This is shown in Figure 11. The BMU cluster weight vector is

$$
W_{29}=(0.873,0.373,0.731,0.065,0.832)
$$

Only one training input vector (part No.35, see Table 2) has been mapped onto cluster \#29. The distance between the new part B and its BMU is 1.2209 and the similarity index is calculated to be at a very low level (24.6\%). 


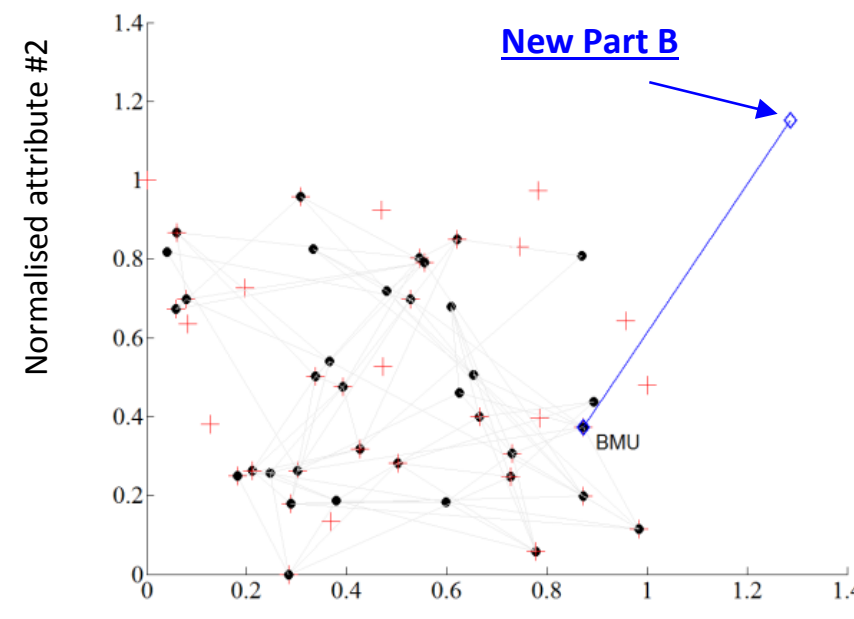

Normalised attribute \#1

Figure 11: New Part B and its BMU cluster (\#29) in the two-dimensional space of attributes \#1 and \#2.

The SOM classifier identified the BMU on the feature map, respectively the most similar previously qualified part(s), for each of the demonstrated new parts, A and B. An additional acceptance criterion for the identified similarity can be utilised using the Similarity Index $S /$ index along with a respective Threshold Limiting Value (TLV). In this study and in the context of the refinishing process application, the choice of the threshold value is guided from the results of validated numerical simulations. The modelling results are used to evaluate the sensitivity of the delamination driver parameter, the stress intensity at the dipped leads interface to package moulding compound, to changes in values of the similarity attributes (see the results summarised in the last column of Table 1). Under the assumption for cumulative linear effects of attributes' changes on the stress intensity at the potential failure site of the components, these model predictions can be used to make an approximate estimation of an upper limit of the difference in the stress intensity between two components. In the context of this demonstration, the $S /$ threshold value criterion aims to prevent accepting the SOM result for the BMU identified for the new part if the stress difference evaluated in such way is above a certain stress limiting value $\left(S_{\text {limit }}\right)$. In this study we select $S_{\text {limit }}=0.5 \mathrm{MPa}$ as previous experimental and correlated modelling results on components dipped under accelerated process conditions indicated that stress intensity change of this magnitude should not result in measurable delamination damage difference $[13,21]$.

If we define the vector $\mathbf{d}=\mathbf{x}_{\mathrm{NEW}}-\mathbf{X}_{\mathrm{BMU}}=\left(d_{1}, d_{2}, d_{3}, d_{4}, d_{5}\right)$ where the attribute vector of the new part is $\mathbf{X}_{\mathrm{NEW}}$ and the BMU cluster weight vector is $\mathbf{X}_{B M U}$ then the $D_{B M U}$ in the definition of the $\mathrm{SI}$ (Eq. 4) would be computed, using Euclidean distance measure, as

$$
D_{B M U}=\sqrt{\sum_{i=1}^{5} d_{i}{ }^{2}}
$$

Based on the assumption for linear cumulative effect of attribute changes on the resulting stress, the TLV is derived so that if the new part and its BMU are such that for cases satisfying the condition

$$
\sum_{i=1}^{5} d_{i} \Delta S_{i}>S_{\text {limit }}
$$

the respective $S /$ index will always be below the TSV. As with the definition in Table $1, \Delta S_{i}$ for $i=1,5$ is the signed value of maximum change in stress intensity for attribute with index $i$ over respective 
range in the dataset for SOM (low to high level). Using Equations 4 and 5, it can be shown that TLV set as

$$
\operatorname{TLV}(\%)=\left(1-\frac{S_{\text {limit }}}{S_{\max } D_{B M U} \sqrt{n}}\right) 100
$$

where $S_{\max }=\max \left\{\left|\Delta S_{i}\right|, i=1,5\right\}$, will ensure that BMU will be rejected as being similar enough to the new part if the condition in Eq. 5 is true. In the above equation $n$ denotes the number of attributes ( $n=5$ in the study case). For the presented demonstration study and the constructed SOM $\left(D_{B M U}=1.62, S_{\max }=2.46 \mathrm{MPa}, S_{\text {limii }}=0.5 \mathrm{MPa}\right.$ and $\left.n=5\right)$, Equitation 6 gives TLV of approximately $94 \%$.

It should be noted that in this instance the TLV value is derived to be very conservative.

Returning back to the similarity results for the demonstrated parts $A$ and $B$, the $S /$ index value for Part $B$ is found to be below the above set threshold limiting value to accept sufficient similarity with the respective $\mathrm{BMU}$; hence the new part $\mathrm{B}$ can be considered for qualification through the defined qualification test procedure as oppose to using similarity criterion. In practice, the similarity results should be used along with other information and knowledge to support the decision for how the qualification should be carried out.

\section{WEIGHTING OF SIMILARITY ATTRIBUTES}

The refinishing process demonstration study for similarity based qualification of electronics parts presented in the paper has adopted the use of the Self-Organising Map algorithm assuming that selected model inputs (i.e. the attributes) have the same significance in relation to the classification target. For example, in the context of the discussed application the interest would be to classify the electronics components as similar if they have similar risk of thermo-mechanical damage when exposed to hot solder dip loads. It is possible that in real applications some attributes may be more indicative for the observed behaviour or the response to a test or load condition than others. In this particular example, although each vulnerability parameter indicates susceptibility to damage of a part subjected to hot solder dip load, different parameters have different indicative capability for the possible damage risks. This relative difference in importance of attributes needs to be captured as part of the similarity classification process.

The way to account for difference in relative importance of attributes, i.e. their weighting on the level of identified similarity, is to use appropriate weights $\left(s_{i}\right)$ for relative importance of attributes, as formulated with the algorithm's pseudo-code in Figure 2.

The step-based procedure detailed in Table 3 can be used to compute the relative weights for the similarity attributes required to run the SOM clustering algorithm. This assumes qualification required is in relation to the risks of thermo-mechanical damage, as with the hot solder dip application.

Table 3: Procedural steps for assigning weights to similarity attributes in model-driven similaritybased qualification of electronic parts

\begin{tabular}{|l|l|}
\hline Step 1 & $\begin{array}{l}\text { Develop parameterised thermo-mechanical finite element models of selected components }(p=1, \\
\left.N_{p}\right) \text { of a particular part constructional class of interest (e.g. QFP, SOT, etc.) that can enable } \\
\text { parametric study for the "similarity attribute" parameters. Use model predictions for a thermo- } \\
\text { mechanical response of interest }(j) \text { obtained from the parametric studies for each modelled } \\
\left.\text { component }(p) \text { to evaluate expected variation (as absolute value, } r_{i j}^{p}\right) \text { in the thermo-mechanical } \\
\text { response } j \text { over the range of interest for the part attribute (PA) } i \text { for component } p . \\
\text { The following notations are assumed: } \\
i \quad \text { - index over component attributes (consider vulnerability parameters as attributes) } \\
\quad N_{A P} \text { - number of attributes }\end{array}$ \\
\hline
\end{tabular}




\begin{tabular}{|c|c|}
\hline & $\begin{array}{cl}j & \text { - index over thermo-mechanical responses } \\
N_{p} & - \text { number of modelled components of a defined part constructional class } \\
r_{i j}^{p} & -j \text {-th response variation over } i \text {-th PA range for component } p\end{array}$ \\
\hline Step 2 & $\begin{array}{l}\text { Using the model predicted values for } r_{i j}^{p} \text { from Step } 1 \text {, for each package response } \boldsymbol{j} \text { and for each } \\
\text { part attribute PA } \boldsymbol{i} \text {, calculate the mean value }\left(\boldsymbol{\mu}_{i j}\right) \text { of the response variation: } \\
\mu_{i j}=\left(\sum_{p=1}^{N_{p}} r_{i j}^{p}\right) / N_{p}\end{array}$ \\
\hline Step 3 & $\begin{array}{l}\text { For each response with index } \boldsymbol{j} \text { find the level of importance } f_{i j} \text { for each part attribute PA with } \\
\text { index } \boldsymbol{i} \text { in relation to the classification criterion }\left(\boldsymbol{f}_{i j} \text { is a relative measure for the extent to which a }\right. \\
\text { response } \boldsymbol{j} \text { is affected by different PAs }) \text { : } \\
f_{i j}=\mu_{i j}\left(\sum_{k=1}^{N_{p_{4}}} \mu_{k j}\right)\end{array}$ \\
\hline Step 4 & $\begin{array}{l}\text { Calculate the relative weight }\left(\boldsymbol{f}_{i}\right) \text { of a part attribute with index } i \text { and use as weight }\left(s_{i}\right) \text { in } \\
\text { the SOM algorithm: } \\
s_{i}=f_{i}=\frac{\sum_{j} f_{i j}}{\sum_{k=1}^{N_{p_{4}}} \sum_{j} f_{k j}}\end{array}$ \\
\hline
\end{tabular}

\section{CONCLUSIONS}

A similarity analysis approach with capability for efficient and accurate classification of electronic components based on selected attributes has been researched and demonstrated. By integrating several similarity concepts originating in feature/geometric similarity models and modern computational intelligence classifiers, a novel approach for similarity-based reliability qualification of electronics components is proposed. The significance of this work is in its potential to transform the way current qualification programmes are undertaken by electronics manufacturers. The main advantage of the proposed approach is in the capability to offer substantial reduction in qualification test times and amount of tested products, and thus reduction of the overall costs of physical testing. Similarity in the case of electronic components demands predominantly constructional and material similarities as these are expected to result in similar outcomes for given process conditions/ qualification physical test specification for observed failure mechanisms. In the context of reliability qualification, the natural choice of similarity attributers is for component characteristics that indicate the susceptibility of the part to anticipated failure modes and damage mechanisms.

The proposed similarity-based approach has been demonstrated on a specific application refinishing electronic components by means of hot solder dipping. In relation to this application, the definition of similarity attributes in the form of vulnerability parameters indicative for the potential risks of thermo-mechanical damage and similarity index are both novel. The study cases are formulated to allow rigorous evaluation, demonstration and testing of the computational approach. The developed methodology and the algorithms used to carry out the demonstration of the proposed qualification method are clearly articulated. The results show extreme efficiency and robustness of classification framework in terms of its capability to perform multi-attribute similarity classification. The proposed similarity-based classifier can be easily adapted and changed to reflect new knowledge, for example new additional attributes can be included to define extra features of the electronic components, etc. Advantages of the proposed algorithm include also the ability of the neural network model to learn and self-adapt as new test and qualification data becomes available. The practical use of the similarity-based qualification approach within an actual qualification programme would require engineering judgement where and how this approach can be used as well 
as knowledge about the required application-specific similarity thresholds and the associated criteria.

\section{ACKNOWLEDGMENTS}

Authors acknowledge the contributions made by Selex ES, Micross Components Ltd, Cassidian (Airbus Defence and Space) and Rolls Royce. We thank John Roulston (Scimus Solutions Ltd.) and Paul Stewart (Selex ES) for the valuable discussions and their support for this research work.

\section{REFERENCES}

[1] C.A. Preussger, R. Blish, S. Huber, T. Dellin and N. Lycoudes (2004) "Understanding and Developing Knowledge-based Qualifications of Silicon Devices", Technology Transfer \#04024492A-TR International SEMATECH, available at:

http://www.sematech.org/docubase/document/4492atr.pdf

[2] A. Engelbrecht (2007) "Computational Intelligence: and introduction", 2nd edition, John Wiley \& Sons Ltd

[3] S. Sumathi and P. Sureckha (2010) "Computational Intelligence Paradigms: Theory and applications using MATLAB", CRC Press

[4] H. K. Lam, S. S. H. Ling, H. T. Nguyen (editors) (2012) "Computational Intelligence and Its Applications", Publ. Imperial College Press

[5] W. Duch (2000) "Similarity-based methods: a general framework for classification, approximation and association", Control and Cybernetics, vol. 29, No. 4, pp. 937-968

[6] T. Kohonen et al. (1996) "Engineering applications of the self-organizing map", Proceedings of the IEEE, Vol. 84 (10), pp. 1358-1384

[7] A. H. de Souza Junior, F. Corona and G. A. Barreto (2013) "Robust Regional Modeling for Nonlinear System Identification using Self-Organizing Maps", In "Advances in Self-Organizing Maps", P.A. Estevez, J. C. Principe, P. Zegers (editors) (2013), Publ. Springer-Verlag, pp. 215224

[8] J. J. Fuertes et al. (2010) "Visual dynamic model based on self-organizing maps for supervision and fault detection in industrial processes", Journal Engineering Applications of Artificial Intelligence, Vol. 23 (1), pp. 8-17

[9] W.D. van Driel, G.Q. Zhang, L. J. Ernst (2005) "Structural Similarity as a Method to Reduce Qualification Tests", Proceedings of 6th International Conference on Electronic Packaging Technology, pp. 1-6

[10] W.D. van Driel, A. Mavinkurve, M.A.J. van Gils, G.Q. Zhang, D.G. Yang, L.J. Ernst (2005) "MultiPhysics Based Structural Similarity Rules for the BGA Package Family", 2005 Electronic Components and Technology Conference, pp. 165-171

[11] W.D. van Driel, A. Mavinkurve, M.A.J. van Gils, G.Q. Zhang (2007) "Advanced structural similarity rules for the BGA package family", Microelectronics Reliability, 47, 205-214

[12] T. Kohonen (2000) Self-organizing maps, Springer

[13] Project Report (2014) "Technology Assessment on the Effects of Refinishing Lead-Free Microelectronic Components", University of Greenwich, UK

[14] J. Vesanto, J. Himberg, E. Alhoniemi and J. Parhankangas, "SOM Toolbox for Matlab 5", Report A57, 2000, available at : http://www.cis.hut.fi/projects/somtoolbox 
[15] J. Vesanto et al (1999) "Self-organizing map in Matlab: the SOM Toolbox", Proceedings of the Matlab DSP Conference, Espoo, Finland, Nov. 16-17, pp. 35-40

[16] Micross Components Ltd, http://www.micross.com

[17] S. Stoyanov et al (2013) "Modelling methodology for thermal analysis of hot solder dip process", Microelectronics Reliability, Vol. 53, pp. 1055-1067

[18] C. Bailey et al (2013) "Assessment of Refinishing Processes for Electronic Components in High Reliability Applications", Proceedings of EPTC, Singapore, Dec. 2013, pp. 156-161

[19] GEIA-STD-0005-2, Standard for Mitigating the Effects of Tin Whiskers in Aerospace and High Performance Electronic Systems, 2005.

[20] C. Yin, C. Best, C. Bailey, S. Stoyanov (2015) "Statistical Analysis of the Impact of Refinishing Process on Leaded Components", Microelectronics Reliability, Vol. 55, Issue 2, pp. 424-431

[21] S. Stoyanov, P. Stewart and C. Bailey (2016) "Vulnerability Study of Hot Solder Dipped COTS Components ", Proceedings of 39th International Spring Seminar on Electronics Technology (ISSE-2016), 18-22 May, Pilsen, Czech Republic, pp. 193-198 